MATHEMATICS OF COMPUTATION

Volume 70, Number 235, Pages 1195-1204

S 0025-5718(00)01241-2

Article electronically published on March 24, 2000

\title{
ERROR ANALYSIS FOR THE COMPUTATION OF ZEROS OF REGULAR COULOMB WAVE FUNCTION AND ITS FIRST DERIVATIVE
}

\author{
YOSHINORI MIYAZAKI, YASUSHI KIKUCHI, DONGSHENG CAI, \\ AND YASUHIKO IKEBE
}

\begin{abstract}
In 1975 one of the coauthors, Ikebe, showed that the problem of computing the zeros of the regular Coulomb wave functions and their derivatives may be reformulated as the eigenvalue problem for infinite matrices. Approximation by truncation is justified but no error estimates are given there.

The class of eigenvalue problems studied there turns out to be subsumed in a more general problem studied by Ikebe et al. in 1993, where an extremely accurate asymptotic error estimate is shown.

In this paper, we apply this error formula to the former case to obtain error formulas in a closed, explicit form.
\end{abstract}

\section{INTRODUCTION}

The second-order linear differential equation

$$
\frac{\mathrm{d}^{2} w}{\mathrm{~d} \rho^{2}}+\left[1-\frac{2 \eta}{\rho}-\frac{L(L+1)}{\rho^{2}}\right] w=0,
$$

where $\rho>0,-\infty<\eta<\infty$, and $L$ is a nonnegative integer, has two independent solutions defined as Coulomb wave functions, one called the regular Coulomb wave function $w=F_{L}(\eta, \rho)$, and the other the irregular Coulomb wave function $w=$ $G_{L}(\eta, \rho)$ (for more details on $F_{L}(\eta, \rho)$ and $G_{L}(\eta, \rho)$, refer to [2]). Equation (1.1) appears in atomic and nuclear physics, and is obtained when we deal with the scattering problems with charged particles 1 or the separation of Schrödinger's wave equation for a Coulomb force field. One will find that there is abundant literature for the computation of the function value $F_{L}(\eta, \rho)$. Nevertheless, when it comes to the computation of the zeros $\rho$ of $F_{L}(\eta, \rho)$, no previous research but 1$]^{2}$ and [5] was found, according to the authors' investigation.

In 1975 [5], one of the coauthors, Ikebe, showed that the problem of computing the zeros of $F_{L}(\eta, \rho)$ and their derivatives may be reformulated as a matrix

Received by the editor July 27, 1999 .

2000 Mathematics Subject Classification. Primary 34L16.

Key words and phrases. Coulomb wave function, eigenvalue problem for infinite matrices, three-term recurrence relations, error estimate.

${ }^{1}$ In this problem, $L$ represents the orbital angular momentum quantum number, $\eta=Z Z^{\prime} e^{2} / \hbar v$, and $\rho=\mu v r / \hbar$, where $Z e, Z^{\prime} e$ are the charges of the two particles, $v$ is their relative velocity, $r$ is the distance between them, and $\mu$ is the reduced mass.

2 [1] proposes a method for computing the zeros $\rho$ of $F_{0}(\eta, \rho)$, and the first three positive zeros are computed for given $\eta=0.0,0.5, \ldots, 3.0$. However, no error estimate is presented for the approximate zeros. 
eigenvalue problem by rewriting the three-term recurrence relations satisfied by $F_{L}(\eta, \rho)$, which represents a minimal solution of the recurrence relations (a secondorder linear homogeneous difference equation) in the sense of 4]. Here are the main theorems of [5]:

[5, Theorem 2.1] Let $L$ and $\eta$ be given. Then $\rho \neq 0$ is a zero of $F_{L}(\eta, \rho)$ if and only if $1 / \rho$ is an eigenvalue of $\mathbf{T}_{L, \eta}$ defined as follows:

$$
\mathbf{T}_{L, \eta}=\left[\begin{array}{cccc}
-\eta d_{L+1} & e_{L+1} & & \mathbf{0} \\
e_{L+1} & -\eta d_{L+2} & e_{L+2} & \\
& e_{L+2} & -\eta d_{L+3} & \ddots \\
\mathbf{0} & & \ddots & \ddots
\end{array}\right]: \ell^{2} \rightarrow \ell^{2}, \text { with }
$$

$d_{k}=\frac{1}{k(k+1)}(k=1,2, \ldots), \quad e_{k}=\frac{1}{k+1} \sqrt{\frac{(k+1)^{2}+\eta^{2}}{(2 k+1)(2 k+3)}}(k=0,1,2, \ldots)$.

Moreover, one finds that an eigenvector of $\mathbf{T}_{L, \eta}$ corresponding to $1 / \rho$ is a nonzero scalar multiple of

$$
\mathbf{0} \neq \mathbf{u} \equiv\left[u^{(1)}, u^{(2)}, \ldots\right]^{T}=\left[\sqrt{2 L+3} F_{L+1}(\eta, \rho), \sqrt{2 L+5} F_{L+2}(\eta, \rho), \ldots\right]^{T} \in \ell^{2} .
$$

Approximate zeros may be computed by truncation to any degrees of accuracy.

[5, Theorem 3.1] Let $L$ and $\eta$ be given. Then $\rho \neq 0$ is a zero of $F_{L}^{\prime}(\eta, \rho)$ if and only if $1 / \rho$ is an eigenvalue of $\tilde{\mathbf{T}}_{L, \eta}$ defined as follows:

$$
\tilde{\mathbf{T}}_{L, \eta}=\left[\begin{array}{ccccc}
\frac{-\eta}{(L+1)^{2}} & \sqrt{\frac{2 L+1}{L+1}} e_{L} & & & \mathbf{0} \\
\sqrt{\frac{2 L+1}{L+1}} e_{L} & -\eta d_{L+1} & e_{L+1} & & \\
& e_{L+1} & -\eta d_{L+2} & e_{L+2} & \\
& & e_{L+2} & -\eta d_{L+3} & \ddots \\
0 & & & \ddots & \ddots
\end{array}\right]: \ell^{2} \rightarrow \ell^{2}
$$

where the definitions of $d_{k}, e_{k}$ are retained as (1.3). Furthermore, an eigenvector of $\tilde{\mathbf{T}}_{L, \eta}$ corresponding to $1 / \rho$ is a nonzero scalar multiple of

$$
\mathbf{0} \neq \tilde{\mathbf{u}}=\left[\sqrt{L+1} F_{L}(\eta, \rho), \sqrt{2 L+3} F_{L+1}(\eta, \rho), \sqrt{2 L+5} F_{L+2}(\eta, \rho), \ldots\right]^{T} \in \ell^{2} .
$$

Approximate zeros may again be computed by truncation to any degrees of accuracy.

What is missing from these two theorems is the precise error estimation. In fact, the derivation of the explicit error estimates for the numerical procedure in $[5$. Theorem 2.1] and [5, Theorem 3.1] is the concern of this paper. Our main results in this regard are stated in the next section (See Theorem 2.1 and Theorem 2.2 in Section 2].

The derivation of [5. Theorem 2.1] and [5, Theorem 3.1] is nothing but a formal matrix reformulation of the recurrence relations satisfied by $u_{L}=F_{L}(\eta, \rho)$, found in [2, Chapter 14], which are (1.7) and (1.8) below. For our purpose, we need two more recurrence relations (1.5) and (1.6) , also found in [2, Chapter 14] (where " 1 " 
represents the $\rho$-derivative):

$$
\begin{aligned}
L \cdot u_{L}^{\prime} & =\sqrt{L^{2}+\eta^{2}} u_{L-1}-\left(\frac{L^{2}}{\rho}+\eta\right) u_{L}, \\
(L+1) \cdot u_{L}^{\prime} & =\left[\frac{(L+1)^{2}}{\rho}+\eta\right] u_{L}-\sqrt{(L+1)^{2}+\eta^{2}} u_{L+1},
\end{aligned}
$$

and by (1.5), (1.6),

$$
\begin{aligned}
& L \sqrt{(L+1)^{2}+\eta^{2}} u_{L+1}=(2 L+1)\left[\frac{L(L+1)}{\rho}+\eta\right] u_{L}-(L+1) \sqrt{L^{2}+\eta^{2}} u_{L-1}, \\
& \begin{aligned}
(1.8) \quad(L+n+1) \sqrt{(L+n)^{2}+\eta^{2}} u_{L+n-1} \\
-(2 L+2 n+1)\left[\frac{(L+n)(L+n+1)}{\rho}+\eta\right] u_{L+n} \\
+(L+n) \sqrt{(L+n+1)^{2}+\eta^{2}} u_{L+n+1}=0, \quad n=0,1,2, \ldots,
\end{aligned}
\end{aligned}
$$

which is obtained from (1.7) by replacing $L$ by $L+n$.

In [5], the asymptotic behavior of $u_{L+n}$ is also derived from (1.8), using [4] Theorem 2.3]:

$$
\frac{u_{L+n+1}}{u_{L+n}}=\frac{\rho}{2 n}[1+o(1)] \rightarrow 0(n \rightarrow \infty) .
$$

The relation (1.8) is also satisfied by $G_{L}(\eta, \rho)$ (i.e., (1.1) still holds true when we substitute $G_{L}(\eta, \rho)$ for $\left.u_{L}\right)$. The point is that $u_{L}=F_{L}(\eta, \rho)$ represents a minimal solution of (1.8). See Gautschi [4].

In 1993, Ikebe et al. [6] studied a more general problem subsuming the former cases, not only justifying the approximation by truncation but also deriving an asymptotic error formula, and it is this theorem, especially (1.10), that we use in this paper to derive the error estimates in Section 2

[6, Theorems 1.1 and 1.4] Given an infinite complex symmetric tridiagonal matrix

$$
\mathbf{A}=\left[\begin{array}{cccc}
d_{1} & f_{2} & & \mathbf{0} \\
f_{2} & d_{2} & f_{3} & \\
& f_{3} & d_{3} & \ddots \\
\mathbf{0} & & \ddots & \ddots
\end{array}\right],
$$

where $d_{k} \rightarrow 0, f_{k} \rightarrow 0(k \rightarrow \infty)$, and $f_{k} \neq 0(k=2,3, \ldots)$, representing a compact operator in $\ell^{2}$. Let $\mathbf{A}$ have a simple eigenvalue $\lambda \neq 0$, and $\mathbf{0} \neq \chi=$ $\left[\chi^{(1)}, \chi^{(2)}, \ldots\right]^{T} \in \ell^{2}$ denote an eigenvector of $\mathbf{A}$ corresponding to $\lambda$. Under the stated assumptions, we have

(i) (converging theorem) Letting $\mathbf{A}_{n}(n=1,2, \ldots)$ be the $n$-th order principal submatrix of $\mathbf{A}$, and $\lambda_{n}$ be an eigenvalue of $\mathbf{A}_{n}$. Then, taking $\left\{\lambda_{n}\right\}$ properly, we have $\lambda_{n} \rightarrow \lambda$.

(ii) (error formula) Assuming that $\left\{\lambda_{n}\right\}$ is taken in the sense of $(i), \chi^{T} \chi \neq 0$, and $\chi^{(n+1)} / \chi^{(n)}$ is bounded for all sufficiently large $n$, we find the following estimate valid:

$$
\lambda-\lambda_{n}=\frac{f_{n+1} \chi^{(n)} \chi^{(n+1)}}{\chi^{T} \chi}[1+o(1)](n \rightarrow \infty) .
$$


In this theorem, the symbol $\ell^{2}$ denotes the complex Hilbert space

$$
\left\{\left[c_{1}, c_{2}, \ldots\right]^{T}: c_{1}, c_{2}, \ldots \in \mathbf{C}, \sum_{n=1}^{\infty}\left|c_{n}\right|^{2}<\infty\right\}
$$

and $o(1)$ a quantity converging to 0 as $n \rightarrow \infty$, and an eigenvalue $\lambda$ is called simple if only one linearly independent eigenvector and no generalized eigenvectors of rank 2 or more correspond to it. The same notation is used in this paper. Up to the present, this theorem has been well applied to the following computations:

1. Zeros of $J_{\nu}(z)$, the Bessel function of the first kind of order $\nu$ [6],

2. Zeros of $z \cdot\left(\mathrm{d} J_{\nu}(z) / \mathrm{d} z\right)+H J_{\nu}(z)$ (with $H$ constant) [3],

3. Eigenvalues of Mathieu's equation [7],

4. Eigenvalues of a differential equation for the spheroidal wave functions [8].

As seen later, the eigenvalues of $\mathbf{T}_{L, \eta}$ (and $\tilde{\mathbf{T}}_{L, \eta}$, too) are all real and simple, the matrices under consideration being compact, real, symmetric, and tridiagonal, where all super- and subdiagonal elements are nonzero (such a matrix operator is diagonalizable, see 9]) and for any given eigenvalue the corresponding eigenvector is uniquely determined (since super- and subdiagonal elements are nonzero, the recurrence relations yield a unique solution up to constant multiplication).

\section{ERROR FORMULAS AND THEIR PROOFS}

We now state two main theorems of this paper: the error formulas in subsection 2.1 (2.1) (2.4) below), followed by the proofs in subsection 2.2

2.1. Error formulas. First, the error formulas shall be shown. Theorem 2.1] deals with the approximate zero of $F_{L}(\eta, \rho)$, while Theorem 2.2 with $F_{L}^{\prime}(\eta, \rho)$.

Theorem 2.1. For each $k$, let $\mathbf{T}_{L, \eta}^{(k)}$ be the $k$-th principal submatrix of $\mathbf{T}_{L, \eta}$ defined in (1.2). Then, one can choose each $\lambda_{k}$, an eigenvalue of $\mathbf{T}_{L, \eta}^{(k)}$, such that $1 / \lambda_{k} \equiv$ $\rho_{k} \rightarrow \rho$. And the following error estimates (2.1) and the rate of convergence (2.2) are valid:

$\rho-\rho_{k}=-\frac{\sqrt{(L+k+1)^{2}+\eta^{2}}}{L+k+1} \cdot \frac{(L+1)^{2}}{(L+1)^{2}+\eta^{2}} \cdot \frac{F_{L+k}(\eta, \rho) F_{L+k+1}(\eta, \rho)}{F_{L+1}^{2}(\eta, \rho)}[1+o(1)]$,

$$
\left.\frac{\rho-\rho_{k+1}}{\rho-\rho_{k}}=\left(\frac{\rho}{2 k}\right)^{2}[1+o(1)] \text { (they hold as } k \rightarrow \infty\right) \text {. }
$$

Theorem 2.2. For each $k$, let $\tilde{\mathbf{T}}_{L, \eta}^{(k)}$ be the $k$-th principal submatrix of $\tilde{\mathbf{T}}_{L, \eta}$ defined in (1.4). Then, one can choose each $\tilde{\lambda}_{k}$, an eigenvalue of $\tilde{\mathbf{T}}_{L, \eta}^{(k)}$, such that $1 / \tilde{\lambda}_{k} \equiv$ $\tilde{\rho}_{k} \rightarrow \rho$. And the following error estimates (2.3) and (2.4) are valid:

$$
\begin{aligned}
\rho-\tilde{\rho}_{k} & =-\rho^{2} \frac{\sqrt{(L+k)^{2}+\eta^{2}}}{L+k} \frac{F_{L+k-1}(\eta, \rho) F_{L+k}(\eta, \rho)}{\left\{\rho^{2}-2 \eta \rho-L(L+1)\right\} F_{L}^{2}(\eta, \rho)}[1+o(1)] \\
& =\frac{\sqrt{(L+k)^{2}+\eta^{2}}}{L+k} \cdot \frac{F_{L+k-1}(\eta, \rho) F_{L+k}(\eta, \rho)}{F_{L}^{\prime \prime}(\eta, \rho) F_{L}(\eta, \rho)}[1+o(1)], \\
& \frac{\rho-\tilde{\rho}_{k+1}}{\rho-\tilde{\rho}_{k}}=\left(\frac{\rho}{2 k}\right)^{2}[1+o(1)](\text { they hold as } k \rightarrow \infty) .
\end{aligned}
$$


2.2. The proofs of Theorems 2.1$]$ and 2.2 . After the introduction of a few more well-known relations by the Coulomb wave functions, we will show newly found relations which are to help the simplification of error formulas, and the proofs of the theorems. First, Wronskian relations and the concrete form of $F_{L}(\eta, \rho)$ are known [2, Chapter 14]:

$$
\begin{gathered}
F_{L}^{\prime}(\eta, \rho) G_{L}(\eta, \rho)-F_{L}(\eta, \rho) G_{L}^{\prime}(\eta, \rho)=1, \\
F_{L-1}(\eta, \rho) G_{L}(\eta, \rho)-F_{L}(\eta, \rho) G_{L-1}(\eta, \rho)=L\left(L^{2}+\eta^{2}\right)^{-1 / 2} \\
F_{L}(\eta, \rho)=C_{L}(\eta) \rho^{L+1} \sum_{k=L+1}^{\infty} A_{k}^{L}(\eta) \rho^{k-L-1}, \text { with } \\
C_{L}(\eta)=\frac{2^{L} e^{-\frac{\pi \eta}{2}}|\Gamma(L+1+i \eta)|}{\Gamma(2 L+2)} \\
A_{L+1}^{L}=1, A_{L+2}^{L}=\frac{\eta}{L+1}, \\
(k+L)(k-L-1) A_{k}^{L}=2 \eta A_{k-1}^{L}-A_{k-2}^{L}(k>L+2) .
\end{gathered}
$$

Next, newly obtained relations shall be shown.

Lemma 2.3. In general, the following relation holds:

$$
\left(u_{L}^{2}\left(\frac{u_{L+1}}{u_{L}}\right)^{\prime}\right)^{\prime}=\left(u_{L+1}^{\prime} u_{L}-u_{L}^{\prime} u_{L+1}\right)^{\prime}=\frac{2(L+1)}{\rho^{2}} u_{L} u_{L+1} .
$$

Proof. The first equality is obvious. Replacing $L$ by $L+1$ in (1.1), one is given

$$
u_{L+1}^{\prime \prime}+\left[1-\frac{2 \eta}{\rho}-\frac{(L+1)(L+2)}{\rho^{2}}\right] u_{L+1}=0 .
$$

(1.1) $\times u_{L+1}-(2.9) \times u_{L}$ yields

$$
u_{L}^{\prime \prime} u_{L+1}-u_{L+1}^{\prime \prime} u_{L}+\frac{2(L+1)}{\rho^{2}} u_{L+1} u_{L}=0 .
$$

Hence, the second equality also holds, since

$$
u_{L+1}^{\prime \prime} u_{L}-u_{L}^{\prime \prime} u_{L+1}=\left(u_{L+1}^{\prime} u_{L}-u_{L}^{\prime} u_{L+1}\right)^{\prime} .
$$

Lemma 2.4. Let $y(\rho) \equiv(2 L+3) u_{L+1}^{2}+(2 L+5) u_{L+2}^{2}+\cdots=\sum_{i=1}^{\infty}(2 L+2 i+1) u_{L+i}^{2}$. Then

$$
y(\rho)=\rho^{2} \frac{\sqrt{(L+1)^{2}+\eta^{2}}}{L+1}\left(u_{L+1}^{\prime} u_{L}-u_{L}^{\prime} u_{L+1}\right) .
$$

Proof. $\{101.5+1.6)\} \times u_{L}$ gives

$$
(2 L+1) u_{L}^{\prime} u_{L}=\sqrt{L^{2}+\eta^{2}} u_{L-1} u_{L}+\frac{2 L+1}{\rho} u_{L}^{2}-\sqrt{(L+1)^{2}+\eta^{2}} u_{L} u_{L+1} .
$$

Replacing $L$ by $L+1, L+2, \ldots$ and adding both sides of each equation yield

$$
\begin{aligned}
& (2 L+3) u_{L+1}^{\prime} u_{L+1}+(2 L+5) u_{L+2}^{\prime} u_{L+2}+\cdots \\
& \quad=\sqrt{(L+1)^{2}+\eta^{2}} u_{L} u_{L+1}+\frac{1}{\rho}\left\{(2 L+3) u_{L+1}^{2}+(2 L+5) u_{L+2}^{2}+\cdots\right\},
\end{aligned}
$$


or

$$
y^{\prime}(\rho)-\frac{2}{\rho} y(\rho)=2 \sqrt{(L+1)^{2}+\eta^{2}} u_{L} u_{L+1} .
$$

The left-hand side of (2.11) is equal to $\rho^{2}\left(y(\rho) / \rho^{2}\right)^{\prime}$, while the right-hand side turns

$$
\rho^{2} \sqrt{(L+1)^{2}+\eta^{2}}\left(u_{L+1}^{\prime} u_{L}-u_{L}^{\prime} u_{L+1}\right)^{\prime} /(L+1)
$$

by (2.8). Equating them gives

$$
\frac{y(\rho)}{\rho^{2}}-\frac{\sqrt{(L+1)^{2}+\eta^{2}}}{L+1}\left(u_{L+1}^{\prime} u_{L}-u_{L}^{\prime} u_{L+1}\right)=c \text { (constant). }
$$

What is left now is to show $c=0$. Consider the asymptotic behavior of the lefthand side of (2.12) as $\rho \rightarrow 0$. Equation (2.7) informs that $u_{L}$ is a power series with its initial term $C_{L}(\eta) \rho^{L+1}$. That means the order of $u_{L+1}^{\prime} u_{L}-u_{L}^{\prime} u_{L+1}$ is at least $O\left(\rho^{2 L+2}\right)$. On the other hand, $y(\rho) / \rho^{2}=O\left(\rho^{2 L+2}\right)$, directly from the definition of $y(\rho)$. Consequently, the conceivable least order of the left-hand side of (2.12) is $O\left(\rho^{2 L+2}\right)$. Since $L \geq 0$, the left-hand side of $(2.12) \rightarrow 0(\rho \rightarrow 0)$. Therefore, $0=c$.

Finally, we are ready to proceed to the proofs of Theorems 2.1 and 2.2 .

Proof for Theorem 2.1. Let us first show that the eigenvalue problem in question satisfies the conditions imposed on [6, Theorems 1.1 and 1.4, part $(i)$ ]. The form of $\mathbf{T}_{L, \eta}$ obviously meets the requirements since $d_{k} \rightarrow 0, e_{k} \rightarrow 0(k \rightarrow \infty)$ and $e_{k} \neq 0$ $(k=0,1,2, \ldots)$. We only have to show that all the eigenvalues of $\mathbf{T}_{L, \eta}$ are simple. In order to prove this, the following two facts are enough, since they are the definition of an eigenvalue being simple in themselves.

- There are no generalized eigenvectors of rank 2 or more corresponding to eigenvalues for an infinite real symmetric matrix in the Hilbert space (see standard books on functional analysis, e.g., [9]).

- Once the first component of an eigenvector of $\mathbf{T}_{L, \eta}$ is given, all the others are uniquely determined, since $e_{k} \neq 0$. That is, there is only one linearly independent eigenvector.

The derivation of an error estimate from [6] Theorems 1.1 and 1.4, part (ii)] follows. First, let us evaluate $\mathbf{u}^{T} \mathbf{u}$. Using $y(\rho)$ defined in Lemma 2.4 we have $\mathbf{u}^{T} \mathbf{u}=y(\rho)$, and

$$
\begin{aligned}
\mathbf{u}^{T} \mathbf{u}= & \rho^{2} \frac{\sqrt{(L+1)^{2}+\eta^{2}}}{L+1}\left(-u_{L}^{\prime} u_{L+1}\right)\left(\text { by } u_{L}=0\right) \\
= & \rho^{2} \frac{(L+1)^{2}+\eta^{2}}{(L+1)^{2}} u_{L+1}^{2} \\
& \left(u_{L}^{\prime}=-\frac{\sqrt{(L+1)^{2}+\eta^{2}}}{L+1} u_{L+1} \text { is given by (1.6) and } u_{L}=0\right) .
\end{aligned}
$$

Next, let us check the conditions. By (2.6),$u_{L+1} \neq 0$ when $u_{L}=0$, leading $\mathbf{u}^{T} \mathbf{u} \neq 0$. And it is obvious by (1.9) that $u^{(n+1)} / u^{(n)}$ is bounded for all sufficiently large $n$. Now that all are cleared, one can put the components of $\mathbf{T}_{L, \eta}, \mathbf{u}$ and (2.13) into (1.10) and obtain (2.1). Equation (2.2) is easily derived by (2.1) and (1.9). 
Proof for Theorem [2.2. Let us skip the proof for [6, Theorems 1.1 and 1.4, part (i)], since they are shown in nearly the same way as Theorem [2.1. Let an error estimate be derived instead. Substituting (1.10) with the components of $\tilde{\mathbf{T}}_{L, \eta}$ and $\tilde{\mathbf{u}}$, one obtains

$$
\rho-\tilde{\rho}_{k}=-\rho^{2} \cdot \frac{\sqrt{(L+k)^{2}+\eta^{2}}}{L+k} \cdot \frac{u_{L+k-1} u_{L+k}}{\tilde{\mathbf{u}}^{T} \tilde{\mathbf{u}}}[1+o(1)](k \rightarrow \infty) .
$$

In order to achieve an error estimate in a closed form (2.3), what is still to be proved is

$$
\tilde{\mathbf{u}}^{T} \tilde{\mathbf{u}}=\left\{\rho^{2}-2 \eta \rho-L(L+1)\right\} u_{L}^{2}\left(=-\rho^{2} u_{L}^{\prime \prime} u_{L}\right) .
$$

By the definition of $\tilde{\mathbf{u}}$ and Lemma 2.4,

$$
\tilde{\mathbf{u}}^{T} \tilde{\mathbf{u}}=(L+1) u_{L}^{2}+y(\rho)=(L+1) u_{L}^{2}+\rho^{2} \frac{\sqrt{(L+1)^{2}+\eta^{2}}}{L+1} u_{L+1}^{\prime} u_{L}\left(\text { by } u_{L}^{\prime}=0\right) \text {. }
$$

Replacing $L$ by $L+1$ in (1.5), which gives

$$
(L+1) \cdot u_{L+1}^{\prime}=\sqrt{(L+1)^{2}+\eta^{2}} u_{L}-\left(\frac{(L+1)^{2}}{\rho}+\eta\right) u_{L+1},
$$

and putting $u_{L}^{\prime}=0$ into (1.6), which also gives

$$
\left(\frac{(L+1)^{2}}{\rho}+\eta\right) u_{L}=\sqrt{(L+1)^{2}+\eta^{2}} u_{L+1},
$$

yields, with $L+1 \geq 1$,

$$
u_{L+1}^{\prime}=\frac{(L+1) u_{L}}{\sqrt{(L+1)^{2}+\eta^{2}}}\left\{1-\frac{2 \eta}{\rho}-\frac{(L+1)^{2}}{\rho^{2}}\right\} .
$$

Substituting this into (2.15), one finally obtains (2.14) (the second equality is simply by (1.1)).

The proof of $\tilde{\mathbf{u}}^{T} \tilde{\mathbf{u}}=(L+1) u_{L}^{2}+y(\rho) \neq 0$ has no difficulty since $y(\rho) \geq 0$ and $(L+1) u_{L}^{2}>0\left(u_{L} \neq 0\right.$ by (2.5) $)$. Equation (2.4) is derived directly by the error estimate (2.3) and (1.9).

\section{NUMERICAL EXPERIMENTS}

We executed the numerical experiments for the presented methods in Theorems 2.1 and 2.2. The computations were done on a Hitachi parallel computer SR2001, using double-precision floating-point arithmetic by FORTRAN77 3 We used the FORTRAN subroutine COMQR 4 in EISPACK [10] for the computation of eigenvalues.

We first computed $\rho_{m}\left(\tilde{\rho}_{m}\right)$ by sufficiently large $m$-th order principal submatrix of (1.2) (1.4) ), and regarded as the true value $\rho$. Then, for each $k$, we computed the reciprocals of all the eigenvalues of $\mathbf{T}_{L, \eta}^{(k)}\left(\tilde{\mathbf{T}}_{L, \eta}^{(k)}\right)$ and chose the closest to $\rho$ to be $\rho_{k}\left(\tilde{\rho}_{k}\right)$. The values of $u_{L+n}(n=0,1,2, \ldots)$ were obtained by back-substitution 5

\footnotetext{
${ }^{3}$ The experiments in this paper, however, do not include parallel computations.

${ }^{4} \mathrm{~A}$ subroutine IMTQL1 in the same package is replaceable, as was used in [5].

${ }^{5}$ By 1.8 and the behavior $u_{L+n} \rightarrow 0(n \rightarrow \infty)$, we let $u_{L+N}=0$ and $u_{L+N-1}=\varepsilon(\neq 0, \varepsilon$ shall be taken appropriately so that an overflow does not occur) for sufficiently large $N$, and computed $u_{L+n}(n=N-1, N-2, \ldots, 0)$ successively in decreasing order.
} 
TABLE 1. Actual errors and estimates of (2.1)

Given $L=1, \eta=1.0$, compute $\rho=6.566570903 \ldots$

\begin{tabular}{|c|c|c|}
\hline$k$ & (A.E.) & (T.E.) \\
\hline \hline 8 & $-5.01 E-05$ & $-5.71 E-05$ \\
9 & $-4.93 E-06$ & $-5.47 E-06$ \\
10 & $-3.99 E-07$ & $-4.35 E-07$ \\
11 & $-2.72 E-08$ & $-2.92 E-08$ \\
12 & $-1.58 E-09$ & $-1.68 E-09$ \\
13 & $-7.94 E-11$ & $-8.36 E-11$ \\
14 & $-3.49 E-12$ & $-3.65 E-12$ \\
\hline
\end{tabular}

TABLE 2. Actual errors and estimates of (2.3)

Given $L=0, \eta=0.0$, compute $\rho=\pi / 2$

\begin{tabular}{|c|c|c|}
\hline $\mathrm{k}$ & (A.E.) & (T.E.) \\
\hline \hline 2 & $-1.03 E-01$ & $-8.75 E-02$ \\
3 & $-6.58 E-03$ & $-6.93 E-03$ \\
4 & $-2.78 E-04$ & $-2.90 E-04$ \\
5 & $-7.36 E-06$ & $-7.56 E-06$ \\
6 & $-1.32 E-07$ & $-1.34 E-07$ \\
7 & $-1.72 E-09$ & $-1.74 E-09$ \\
8 & $-1.70 E-11$ & $-1.71 E-11$ \\
\hline
\end{tabular}

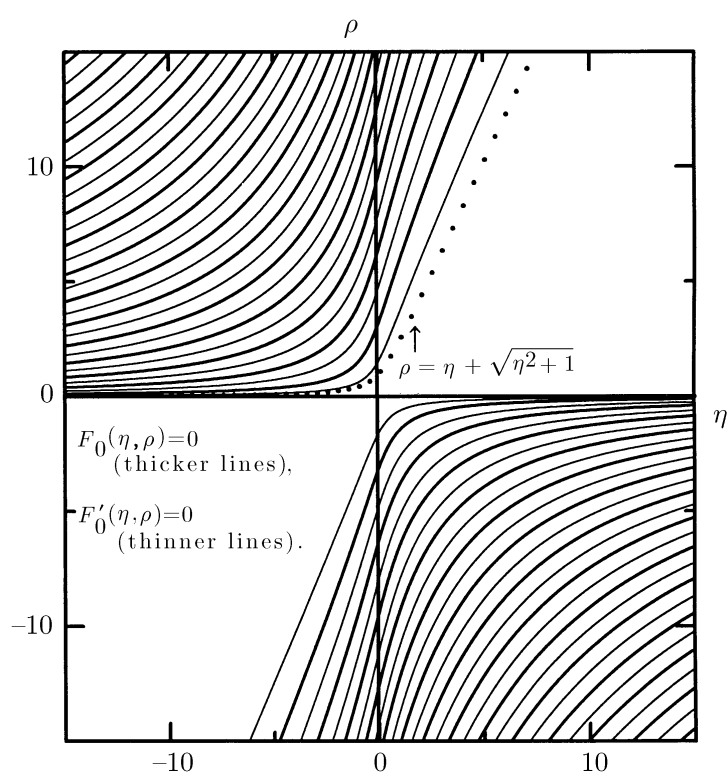

Figure 1. $(\eta, \rho)$-pairs satisfying $F_{0}(\eta, \rho)=0, F_{0}^{\prime}(\eta, \rho)=0$ and $\rho=\eta+\sqrt{\eta^{2}+1}$ 
Table 1 is the result of the numerical computations for a zero of $F_{L}(\eta, \rho)$, and Table 2 of $F_{L}^{\prime}(\eta, \rho)$. In the tables, actual errors (A.E.) represent the left-hand side of (2.1) $((2.3))$ divided by $\rho$ while theoretical errors (T.E.) represent the right-hand side of ([2.1) $([2.3))$ without $[1+o(1)]$, again divided by $\rho$, and 3 significant figures are displayed after rounding. One can observe that A.E. and T.E. get closer and each error gets smaller acceleratively as $k$ becomes larger. Those figures are in agreement for the first digit in Table 1 and for the first two digits in Table 2 ,

Let us show another result in Figure 1, or the $(\eta, \rho)$-plots satisfying $F_{0}(\eta, \rho)=$ 0 and $F_{0}^{\prime}(\eta, \rho)=0$. For each $\eta$, the computation of $\rho$ was performed by the stated procedure. To visualize (4.1) (appearing in the next section), $\rho=\eta+$ $\sqrt{\eta^{2}+(L+1)^{2}}$ (with $\left.L=0\right)$ is also plotted.

\section{Miscellaneous: Remarks on the zeros of $F_{L}(\eta, \rho)$ and $F_{L}^{\prime}(\eta, \rho)$}

This final section focuses on some remarks on the zeros $\rho$ of $F_{L}(\eta, \rho)$ and $F_{L}^{\prime}(\eta, \rho)$.

Remark 4.1. For given $L$ and $\eta$, the region of zeros of $F_{L}^{\prime}(\eta, \rho)$ is determined by the inequality

$$
\rho>\eta+\sqrt{\eta^{2}+(L+1)^{2}} .
$$

Proof. By 2.14), $u_{L}^{2}\left\{\rho^{2}-2 \eta \rho-(L+1)^{2}\right\}=(2 L+3) u_{L+1}^{2}+(2 L+5) u_{L+2}^{2}+\cdots$. The right-hand side is obviously positive, then so is the left-hand side. That means $\rho^{2}-2 \eta \rho-(L+1)^{2}>0$. Considering $\rho>0$, one has $\rho>\eta+\sqrt{\eta^{2}+(L+1)^{2}}$.

Remark 4.2. For given $L$ and $\eta$, the region of zeros of $F_{L}(\eta, \rho)$ is also confined to (4.1).

Proof. Denoting the smallest zero of $u_{L}\left(u_{L}^{\prime}\right)$ by $\rho_{0}^{1 \text { st }}\left(\rho_{1}^{1 \text { st }}\right)$, we will show $\rho_{1}^{1 \text { st }}<\rho_{0}^{1 \text { st }}$. Noting that $u_{L} \rightarrow 0$ as $\rho \rightarrow 0$ (by the form of $u_{L}$ in (2.7) ) and $u_{L}\left(\eta, \rho_{0}^{1 \text { st }}\right)=0$, one finds, from Rolle's theorem, that there exists at least one $\rho$ satisfying $u_{L}^{\prime}=0$ in $\left(0, \rho_{0}^{1 \text { st }}\right)$. Therefore, $\rho_{1}^{1 \text { st }}<\rho_{0}^{1 \text { st }}$. This and Remark 4.1 are sufficient to prove the proposition.

Remark 4.3. There is one and only one zero of $F_{L}^{\prime}(\eta, \rho)$ between two continuous zeros of $F_{L}(\eta, \rho)$.

Proof. Let us prove that "there is one and only one zero of $F_{L}(\eta, \rho)$ between two continuous zero of $F_{L}^{\prime}(\eta, \rho)$ ", which is equivalent to the proposition. By (2.14), $\rho^{2}-2 \eta \rho-L(L+1)>0$ holds when $u_{L}^{\prime}=0$. Recalling (1.1), which is $u_{L}^{\prime \prime}+$ $\left\{\rho^{2}-2 \eta \rho-L(L+1)\right\} u_{L} / \rho^{2}=0$, we find that $u_{L}$ and $u_{L}^{\prime \prime}$ have different signs then. Also note $u_{L} \neq 0$ (and so is $u_{L}^{\prime \prime}$ ) when $u_{L}^{\prime}=0$ by (2.5).

Suppose $\rho_{1}, \rho_{2}\left(\rho_{1}<\rho_{2}\right)$ are two continuous zeros of $u_{L}$. Then, $u_{L}$ is of definite sign in $\left(\rho_{1}, \rho_{2}\right)$. Now, without the loss of generality, we may assume $u_{L}>0$. If there are more than one zero of $u_{L}^{\prime}$ in $\left(\rho_{1}, \rho_{2}\right)$, there is at least one pair of maximal and minimal points of $u_{L}$ there, which is absurd since $u_{L}^{\prime \prime}>0$ at a minimal point and $u_{L}$ and $u_{L}^{\prime \prime}$ are of the same sign.

\section{REFERENCES}

1. M. Abramowitz, Asymptotic Expansions of Coulomb Wave Functions, MR, Vol. VII, No. 1 (1949), 75-84. MR 10:454a

2. M. Abramowitz and I. A. Stegun, Handbook of Mathematical Functions, Dover, N.Y., (1972). MR 34:8606 
3. N. Asai, Y. Miyazaki, D. Cai, K. Hirasawa, and Y. Ikebe, Matrix Methods for the Numerical Solution of $z J_{\nu}^{\prime}(z)+H J_{\nu}(z)=0$, Electronics and Communications in Japan, Part 3, Vol. 80, No. 7 (1997), 44-54.

4. W. Gautschi, Computational Aspects of Three-Term Recurrence Relations, SIAM Rev., 9 (1967), 24-82. MR 35:3927

5. Y. Ikebe, The Zeros of Regular Coulomb Wave Functions and of Their Derivatives, Math. Comp., 29(131), (1975), 878-887. MR 51:14529

6. Y. Ikebe, Y. Kikuchi, I. Fujishiro, N. Asai, K. Takanashi, and M. Harada, The Eigenvalue Problem for Infinite Compact Complex Symmetric Matrices with Application to the Numerical Computation of Complex Zeros of $J_{0}(z)-i J_{1}(z)$ and of Bessel Functions $J_{m}(z)$ of Any Real Order m, Linear Algebra Appl., 194 (1993), 35-70. MR 94g:47025

7. Y. Miyazaki, N. Asai, D. Cai, and Y. Ikebe, A Numerical Computation of the Inverse Characteristic Values of Mathieu's Equation, Transactions of the Japan Society for Industrial and Applied Mathematics, 8(2), (1998), 199-222 (in Japanese).

8. Y. Miyazaki, N. Asai, D. Cai, and Y. Ikebe, The Computation of Eigenvalues of Spheroidal Differential Equations by Matrix Method, JSIAM Annual Meeting, (1997), 224-225 (in Japanese).

9. F. Riesz and B. S. Nagy, Functional Analysis, Dover, N.Y., (1990). MR 91g:00002

10. B. T. Smith, J. M. Boyle, J. J. Dongarra, B. S. Garbow, Y. Ikebe, V. C. Klema, and C. B. Moler, Matrix Eigensystem Routines - EISPACK Guide, Second Edition, Springer-Verlag, (1976). MR 58:1366a

Faculty of Communications and Informatics, Shizuoka Sangyo University, Surugadai 4-1-1, Fujieda, Shizuoka, 426-8668, Japan

E-mail address: yoshi@fujieda-ssu.ac.jp

Department of Computer Software, The University of Aizu, Tsuruga, Ikkimachi, Aizunakamatsu, Fukushima, 965-8580, Japan

E-mail address: kikuchi@u-aizu.ac.jp

Institute of Information Sciences and Electronics, The University of Tsukuba, Tennodai 1-1-1, Tsukuba, Ibaraki, 305-8573, Japan

E-mail address: cai@is.tsukuba.ac.jp

Department of Computer Software, The University of Aizu, Tsuruga, Ikkimachi, Aizunakamatsu, Fukushima, 965-8580, Japan

E-mail address: ikebe@u-aizu.ac.jp 